\title{
Morphological Determinants of Femoral Strength in Growth Hormone-Deficient Transgenic Growth-Retarded (Tgr) Rats*
}

\author{
BAJ EVANS, ${ }^{1,2}$ JT WARNER, ${ }^{1,2}$ C ELFORD,${ }^{1}$ SL EVANS,${ }^{3}$ A LAIB,${ }^{5}$ RK BAINS, ${ }^{6}$ \\ JW GREGORY, ${ }^{1}$ and T WELLS ${ }^{4}$
}

\begin{abstract}
The extent to which childhood GHD affects adult fracture risk is unclear. We measured femoral strength in adult transgenic growth-retarded rats as a model of GHD. Long-term, moderate GHD was accompanied by endocrine and morphometric changes consistent with a significant reduction in femoral strength.
\end{abstract}

Introduction: Childhood growth hormone deficiency (GHD) is associated with osteopenia, but little is known about its effects on subsequent adult bone strength and fracture risk.

Materials and Methods: We have therefore measured femoral strength (failure load measured by three-point bending) in a new model of moderate GHD, the transgenic growth-retarded (Tgr) rat at 15, 22-23, and 52 weeks of age, and have quantified potential morphological and endocrine determinants of bone strength.

Results: Skeletal growth retardation in Tgr rats was accompanied by a sustained reduction in the anterior-posterior diameter of the femoral cortex, whereas mid-diaphyseal cortical wall thicknesses were largely unaltered. Total femoral strength was significantly impaired in Tgr rats $(p<0.01)$, and this impairment was more pronounced in males than females. Compromised bone strength in Tgr rats could not be accounted for by the reduction in mechanical load (body weight) and was not caused by impairment of the material properties of the calcified tissue (ultimate tensile stress), despite marked reductions in femoral mineral density (areal bone mineral density; $p<0.001$ ). Microcomputerized tomographical analysis revealed significant modification of the architecture of trabecular bone in Tgr rats, with reductions in the number and thickness of trabeculae $(p<0.05)$ and in the degree of anisotropy $(p<0.01)$. The marked reduction in plasma insulin-like growth factor-1 in Tgr rats was accompanied by the development of high circulating leptin levels $(p<0.01)$. Conclusion: These results show that the changes in endocrinology and bone morphology associated with long-term moderate GHD in Tgr rats are accompanied by changes consistent with a significant reduction in the threshold for femoral fracture.

J Bone Miner Res 2003;18:1308-1316

Key words: bone strength, femoral morphology, growth hormone, growth hormone deficiency, leptin, transgenic rats

\section{INTRODUCTION}

$\mathrm{G}$ ROWTH HORMONE $(\mathrm{GH})$ is the primary regulator of postnatal longitudinal bone growth in mammals and also

*Preliminary reports of sections of this work have previously been presented in abstract form at meetings of the British Society for Paediatric Endocrinology and Diabetes (Portsmouth, 1999), the Bone and Tooth Society (Cambridge, 2000), the European Society for Biomechanics (Dublin, 2000), the Growth Hormone Research Society (Göteborg, 2000), and the European Society for Paediatric Endocrinology (Brussells, 2000).

Dr Gregory has received partial funding from Pharmacia Upjohn and Novo Nordisk. All other authors have no conflict of interest. contributes to the humoral control of bone mineralization. ${ }^{(1,2)}$ In humans, adult-onset GH deficiency (GHD) leads to impairment of bone mineral density (BMD) ${ }^{(3)}$ and an increased fracture risk. ${ }^{(4)}$ Childhood GHD, which is often diagnosed with less stringent criteria, ${ }^{(5-7)}$ is also associated with impaired bone mineral accumulation and turnover, ${ }^{8,9)}$ and if uncorrected through puberty, may result in reduced peak bone mass in young adult life. ${ }^{(10,11)}$ The extent to which failure to achieve adequate peak bone mass in late adolescence and early adulthood leads to an increased risk of subsequent osteoporosis and bone fracture remains unclear. $^{(12)}$

\footnotetext{
${ }^{1}$ Department of Child Health, University of Wales College of Medicine, Cardiff, United Kingdom.

${ }^{2}$ These authors contributed equally to this work.

${ }^{3}$ School of Engineering, Cardiff University, Cardiff, United Kingdom.

${ }^{4}$ School of Biosciences, Cardiff University, Cardiff, United Kingdom.

${ }^{5}$ SCANCO Medical AG, Bassersdorf, Switzerland.

${ }^{6}$ Division of Molecular Neuroendocrinology, National Institute for Medical Research, London, United Kingdom.
} 
Because many of the processes of bone growth and remodeling are similar in rats and humans, ${ }^{(13)}$ we have used a new model of moderate GHD, the transgenic growthretarded (Tgr) rat, to examine the effects of reduced circulating $\mathrm{GH}$ on bone strength. In this model, expression of human (h) $\mathrm{GH}$ in the arcuate $\mathrm{GH}$-releasing factor neurons causes a $60 \%$ reduction in pituitary GH content and a $50 \%$ reduction in the amplitude of the episodes of $\mathrm{GH}$ secretion. ${ }^{(14,15)}$ This represents a similar degree of GHD to that seen most frequently in childhood-onset GHD patients and may represent a more appropriate model for analysis of bone strength in this context than the more profoundly deficient $d w / d w$ rat. ${ }^{(16)}$

In the studies reported here, we describe the development of impaired femoral strength with age in both male and female Tgr rats, relating total bone and material strength with endocrine, morphometric, and mineral density parameters. In addition, to overcome the limitations related to measurement of areal mineral density, we used microcomputerized tomographical (micro-CT) scanning to analyze bone density and describe modifications in trabecular architecture in the Tgr model of GHD.

\section{MATERIALS AND METHODS}

\section{Tgr rats}

The animal procedures described conformed to the institutional and national ethical guidelines for experiments with genetically modified rats. Hemizygous Tgr rats and normal wild-type (WT) littermates were derived from the original colony at the National Institute for Medical Research (London, UK) and bred in the Transgenic Unit (School of Biosciences, Cardiff University). All animals were housed under conditions of 14-h light:10-h dark (lights on at 5:00 a.m.), with food (Harlan Teklad Rodent Maintenance Diet, Harlan, UK; nutritional composition: protein $14.2 \%$; oil $4.9 \%$; calcium $0.6 \%$; sodium $0.25 \%$; potassium $0.39 \%$; phosphorous $0.32 \%$; chloride $0.52 \%$ ) and water available ad libitum.

\section{Skeletal growth and weight gain in Tgr rats}

Groups of nonfasting 15-, 22-23-, and 52-week-old male and female WT and Tgr rats $(n=4-6)$ from the Cardiff colony were weighed and killed. Trunk blood was collected, and separated plasma was stored at $-20^{\circ} \mathrm{C}$ for subsequent determination of insulin-like growth factor-1 (IGF-1) and leptin concentrations. Left femurs were dissected, total length was measured with a hand-held micrometer, and the femurs were wrapped in sterile saline gauze and stored at $-20^{\circ} \mathrm{C}$ before measurements of bone mineral content (BMC), strength and morphology, and trabecular organization.

\section{Femoral mineralization in Tgr rats}

Bone mineral content (BMC) was measured by DXA using the Lunar Pixi small animal scanner. The accuracy of this technique in measuring calcium content was confirmed in preliminary studies, in which a highly significant correlation between femoral total BMC and ash weight $(r=0.86$; $p<0.0001$ ) was obtained. ${ }^{(17)}$ The $\mathrm{CV}$ for femoral BMD for five repeated scans of 30 femurs, with repositioning be- tween scans, was $2.7 \%$. Bones were thawed at room temperature for 30 minutes before measurement and aligned anterior-posteriorly relative to the scanning beam. Measurements of total BMC $(\mathrm{g})$ and scanned bone area $\left(\mathrm{BA} ; \mathrm{cm}^{2}\right)$ were made, and areal BMD (aBMD; $\mathrm{g} / \mathrm{cm}^{2}$ ) was calculated as BMC/BA. In addition, femoral neck and distal femur aBMD were determined by measuring BMC in a fixed area $\left(0.03 \mathrm{~cm}^{2}\right)$ of each region of interest.

\section{Femoral strength and morphology in Tgr rats}

Previously frozen left femurs were thawed at room temperature, but only removed from their container just before mechanical testing to maintain moisture content. The bones were loaded in three-point bending between $10 \mathrm{~mm}$ diameter rollers (16 mm apart), with the middle roller $8 \mathrm{~mm}$ from the outer rollers and positioned over the thinnest part of the femoral shaft, level with the distal end of the lateral ridge that runs along the proximal part of the femur toward the greater trochanter. The posterior aspect of the condyles rested on the side of the outer roller, and the bones were orientated such that they were loaded in a roughly posterior direction. Each bone was loaded at a crosshead speed of 2 $\mathrm{mm} /$ minute until failure, with load and displacement data recorded by the computer controlled testing machine (Lloyd LRX tensile testing machine with 100N load cell; Lloyd Instruments, Segensworth, Hants, UK).

Mid-diaphyseal cortical medio-lateral (M-L) and anterior posterior (A-P) diameters, and lateral, medial, anterior, and posterior wall thicknesses were measured at the fracture site using a Vickers microscope and micrometer stage. Using these measurements and simple beam theory, ultimate tensile stress (UTS) was calculated using:

$$
\sigma=M y / I
$$

where the bending moment, $M$, is one-half the applied load multiplied by the distance from the central support, $y$ is one-half the outside depth, and the second moment of area, I, is given by:

$$
I=\frac{\pi}{64}\left(b_{o} d_{o}^{3}-b_{i} d_{i}^{3}\right)
$$

where $b$ and $d$ are the breadth and depth of the crosssection, respectively, and the subscripts $o$ and $i$ indicate the inside and outside dimensions, respectively.

\section{Trabecular organization in Tgr rats}

Bones were scanned with a commercially available highresolution microcomputerized tomography (micro-CT) system ( $\mu$ CT-40; Scanco Medical, Basserdorf, Switzerland). Using this system, three-dimensional (3-D) information is obtained by stacking successively measured slices on top of each other. No sample preparation is required, and because the procedure is nondestructive, the bones remain available for other examination techniques. The chosen voxel size was $12 \mu \mathrm{m}$ in all three spatial dimensions, and for each sample, 178 slices were measured. 3-D visualizations of complete measurements from femurs from WT and Tgr animals are shown in Figs. 5A and 5B. 


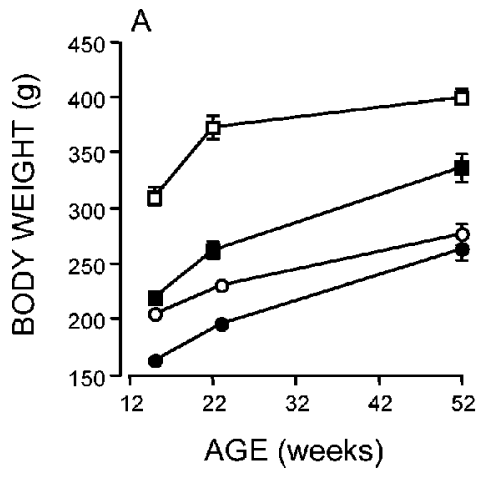

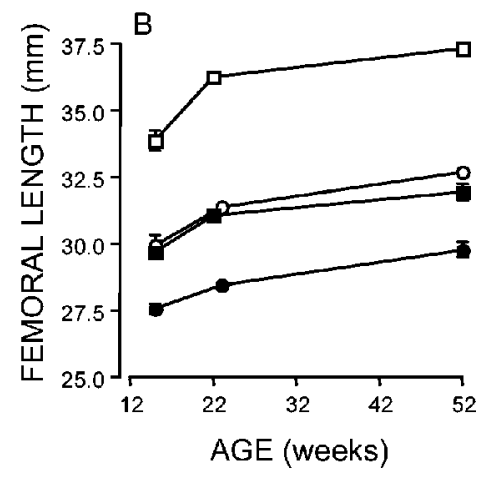

FIG. 1. Developmental growth changes in male (squares) and female (circles) WT (open symbols) and Tgr (closed symbols) rats. Parameters shown are (A) body weight and (B) femoral length. Values shown are mean \pm SE $(n=4-6$; and statistical comparisons described in the text made using one-way ANOVA and Bonferroni comparison).
The trabecular and the cortical part of each distal femur were separated with semi-automatically drawn contours. ${ }^{(18)}$ The complete secondary spongiosa could then be evaluated, and sampling errors brought by random deviations of single section were avoided. The resulting grayscale images were segmented using a low-pass filter to remove noise and a fixed threshold to extract the mineralized bone phase. From the binarized images, structural indices of the appearance of trabecular bone were assessed with recently developed 3-D techniques without model assumptions. Relative bone volume and the number, thickness, and separation of the trabeculae were calculated by measuring 3-D distances ${ }^{(19)}$ directly in the trabecular network and taking the mean over all voxels. The diameter of spheres filling the structure was taken as trabecular thickness, the thickness of the marrow spaces as trabecular separation, and the inverse of the mean distances of the mineralized structure was calculated as trabecular number. Degree of anisotropy was determined using a 3-D method similar to the mean intercept length (MIL), ${ }^{(18)}$ and was taken as the ratio of the biggest MIL divided by the smallest MIL (i.e., a degree of anisoptropy of 1.0 denotes an isotropic structure).

\section{Plasma IGF-1 and leptin concentrations in Tgr rats}

Plasma IGF-1 and leptin concentrations were determined by radioimmunoassay (RIA; Immunodiagnostic Systems Ltd., Boldon, UK and Linco Research Inc., St Charles, MO, USA, respectively). The mean inter- and intra-assay CVs for these assays were $5.7 \%$ and $4.1-9.4 \%$ (IGF-1) and $3.0-5.7 \%$ and $2.0-4.6 \%$ (leptin), respectively.

\section{Statistical analysis}

All data are expressed as mean $\pm \mathrm{SE}$, with statistical comparisons being performed by Student's $t$-test or ANOVA plus Bonferroni/Dunnett's post hoc test as appropriate.

\section{RESULTS}

\section{Skeletal growth and weight gain in Tgr rats}

At the end of the rapid growth phase (15-23 weeks), the lower body weights in Tgr rats (Fig. 1A; $30 \%$ less in males, $20 \%$ less in females; $p<0.001$ ) were accompanied by significantly shorter femoral lengths (Fig. 1B; $13 \%$ less in males, $9 \%$ less in females; $p<0.001)$. During the slower phase of growth (23-52 weeks), body weights in Tgr rats continued to increase at a higher rate than that of their WT littermates $(p<0.001)$, whereas femoral length increased $(p<0.05)$ in Tgr rats parallel with their WT counterparts. This higher rate of body weight gain may be accounted for, in part, by increased adiposity in Tgr rats, visceral fat depots being proportionately larger in Tgr rats than in their WT littermates ( $p<0.01$; data not shown).

\section{Femoral mineralization in Tgr rats}

Total femoral BMC and aBMD increased rapidly in WT males between 15 and 22 weeks (Figs. 2A and 2B; $p<$ 0.01 ), but remained unaltered thereafter. Total femoral $\mathrm{BMC}$ and aBMD were markedly lower in Tgr males (BMC $40 \%$ less; aBMD 20\% less; $p<0.001$ ), but the same developmental pattern was observed. Total femoral BMC continued to rise with age in WT females $(p<0.05)$ and remained 30\% lower than that in WT males $(p<0.01)$. However, total femoral aBMD was only significantly different between WT males and WT females in 22- to 23week-old rats $(p<0.001)$. Femoral BMC and aBMD in Tgr females were significantly lower than WT females at all ages (BMC 30\% less; aBMD 18\% less; $p<0.001$ ), increasing significantly between 23 and 52 weeks $(p<0.01)$. In comparison with Tgr males, femoral BMC in Tgr females was significantly lower at both 22-23 weeks and at 52 weeks $(p<0.001)$, but was only reflected in a significant difference in aBMD at 22-23 weeks $(p<0.001)$.

Analysis of aBMD in different regions revealed that in the femoral neck aBMD increased continuously with age in all groups, showing a $60-80 \%$ increase from 15 to 52 weeks (Fig. 2C; $p<0.001$ ). In contrast, aBMD in the distal femur showed a small decline from 15 to 22 weeks in all but the Tgr males (Fig. 2D; $p<0.05$ ), increasing in all groups from 22 to 52 weeks $(p<0.01)$ to produce a small $(15 \%)$ overall elevation (15-52 weeks) in all but the WT females $(p<0.05)$. In Tgr rats, aBMD was significantly lower in the trabecular neck region of the femur at 52 weeks $(15-25 \%$ less; $p<0.01$ ) and was significantly reduced at all ages in the distal femur (Fig. 2D; 16-24\% less; $p<0.001$ ). There was no observable sexual dimorphism in aBMD in either neck or distal portions of the femur.

\section{Femoral strength and morphology in Tgr rats}

Morphometric analysis of the fracture site after strength testing (discussed below) revealed that mid-diaphyseal cor- 

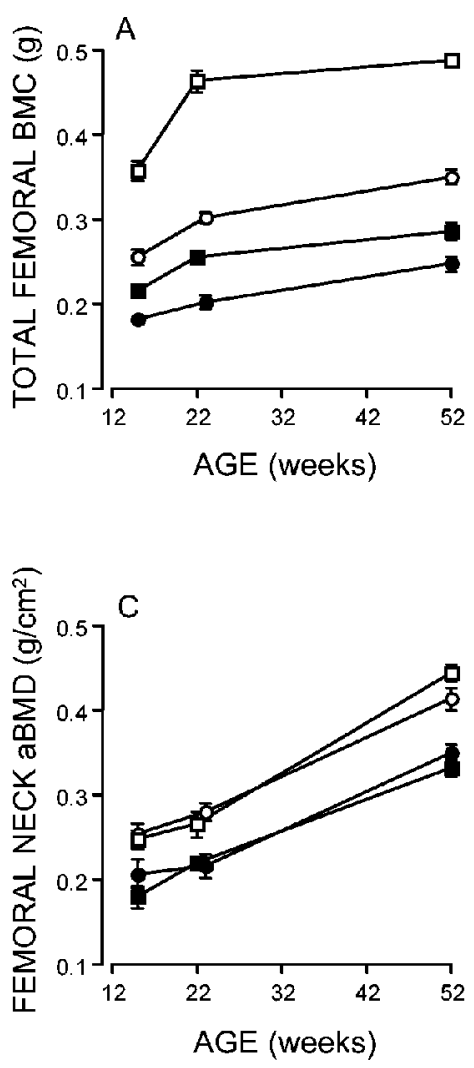
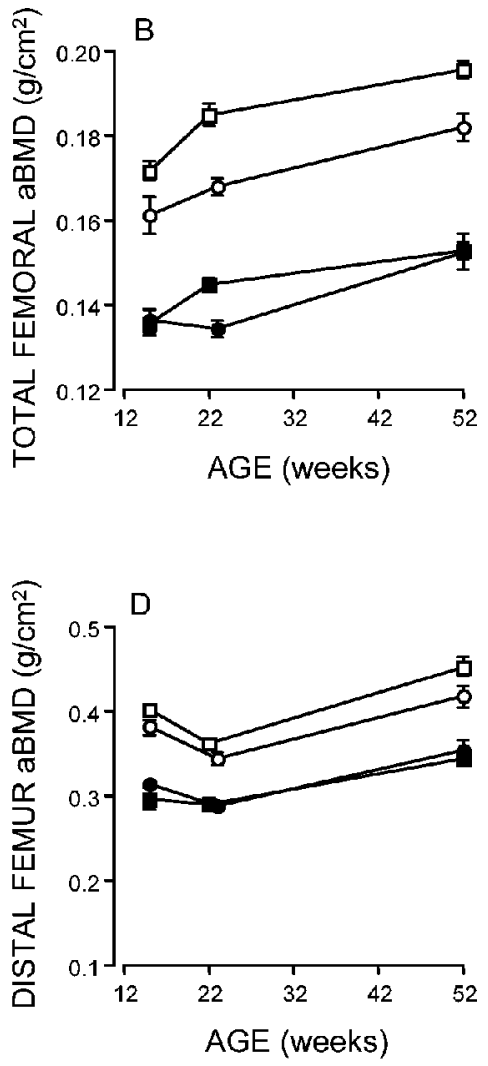

FIG. 2. The development of femoral mineralization in male (squares) and female (circles) WT (open symbols) and Tgr (closed symbols) rats. Parameters shown are (A) total femoral BMC, (B) total femoral aBMD, (C) femoral neck aBMD, and (D) distal femur aBMD. Values shown are mean $\pm \operatorname{SE}(n=4-6$; and statistical comparisons described in the text made using one-way ANOVA and Bonferroni comparison). tical A-P diameters in 15-week-old male Tgr rats were $20 \%$ smaller than in WT males (Figs. 3A and 3B; $p<0.001$ ). This difference $(p<0.01)$ was less pronounced $(10 \%)$ in females. A-P diameters did not change with age. At 15 weeks of age, mid-diaphyseal cortical M-L diameters were significantly greater in WT males than in any other group $(p<0.001)$ and had increased steeply by 22 weeks $(p<$ $0.001)$. By 1 year, mid-diaphyseal cortical M-L diameters had converged in all groups. The sex-dependent differences in femoral diameter in WT rats were not observed in Tgr animals. The pattern of age-dependent changes in lateral and medial mid-diaphyseal cortical wall thicknesses was most pronounced in Tgr rats (Figs. 3E and 3F). Between 15 and 22-23 weeks, lateral wall thickness decreased rapidly in male $(p<0.05)$ and female $(p<0.01)$ Tgr rats, while medial wall thickness increased steeply over the same period (males, $p<0.01$; females, $p<0.001$ ). This pattern was not statistically significant in WT animals. There were no significant differences in mid-diaphyseal cortical anterior and posterior wall thicknesses between the groups, and these parameters did not change with age (Figs. 3C and 3D).

Cortical femoral strength was assessed by two methods: direct measurement of failure load, indicating the strength of the whole bone, and calculation of UTS, an index of the strength of the calcified tissue per se. Although failure load was similar in male and female WT rats at 15 weeks, total bone strength diverged thereafter because of a large (33\%) and sustained elevation in failure load in WT males at 22 weeks (Fig. 4A; $p<0.001$ ). This rapid rise in failure load was not seen in Tgr males, which remained significantly weaker than their WT littermates at all ages $(p<0.01)$ and similar to their female Tgr littermates. These differences in failure load were not reflected in parallel changes in UTS. Indeed, at 15 weeks, UTS was $25 \%$ lower in WT males than any other group (Fig. 4B; $p<0.001$ ). In Tgr males, UTS decreased by $20 \%$ between 15 and 22 weeks, at which age there was a clear sexual dimorphism in the mechanical properties of the calcified material in both strains $(p<$ 0.05). UTS subsequently converged in all groups.

Regression analysis revealed that failure load was positively correlated with body weight in WT males $\left(r^{2}=0.86\right.$; Fig. 4C). This relationship was disrupted in Tgr males $\left(r^{2}=\right.$ $0.10)$ and was not observed in either group of female rats. UTS was not correlated with body weight in any group (data not shown). Adjustment of failure load to correct for the influence of body weight demonstrated that, after the rapid growth phase, Tgr males had significantly weaker bones on a per gram body weight basis than any other group $(p<$ 0.01; Fig. 4D).

\section{Trabecular organization in Tgr rats}

Micro-CT analysis of the trabecular region of a subgroup of femurs from 15-week-old males revealed that femurs from Tgr rats had $32 \%$ less calcified tissue per unit volume (relative bone density) than WT males ( $p<0.01$; Table 1 ; Fig. 5). This was due primarily to a lower number of trabeculae (connectivity density; $19 \%$ less; $p<0.05$ ), with a smaller reduction in trabecular thickness $(11 \%$ thinner; $p<0.05)$ and a corresponding increase in trabecular separation $(p<0.01)$. In addition, trabeculae from Tgr rats had a significantly greater intra-individual range of thicknesses than those in WT femurs ( $p<0.05$; data not shown), and 

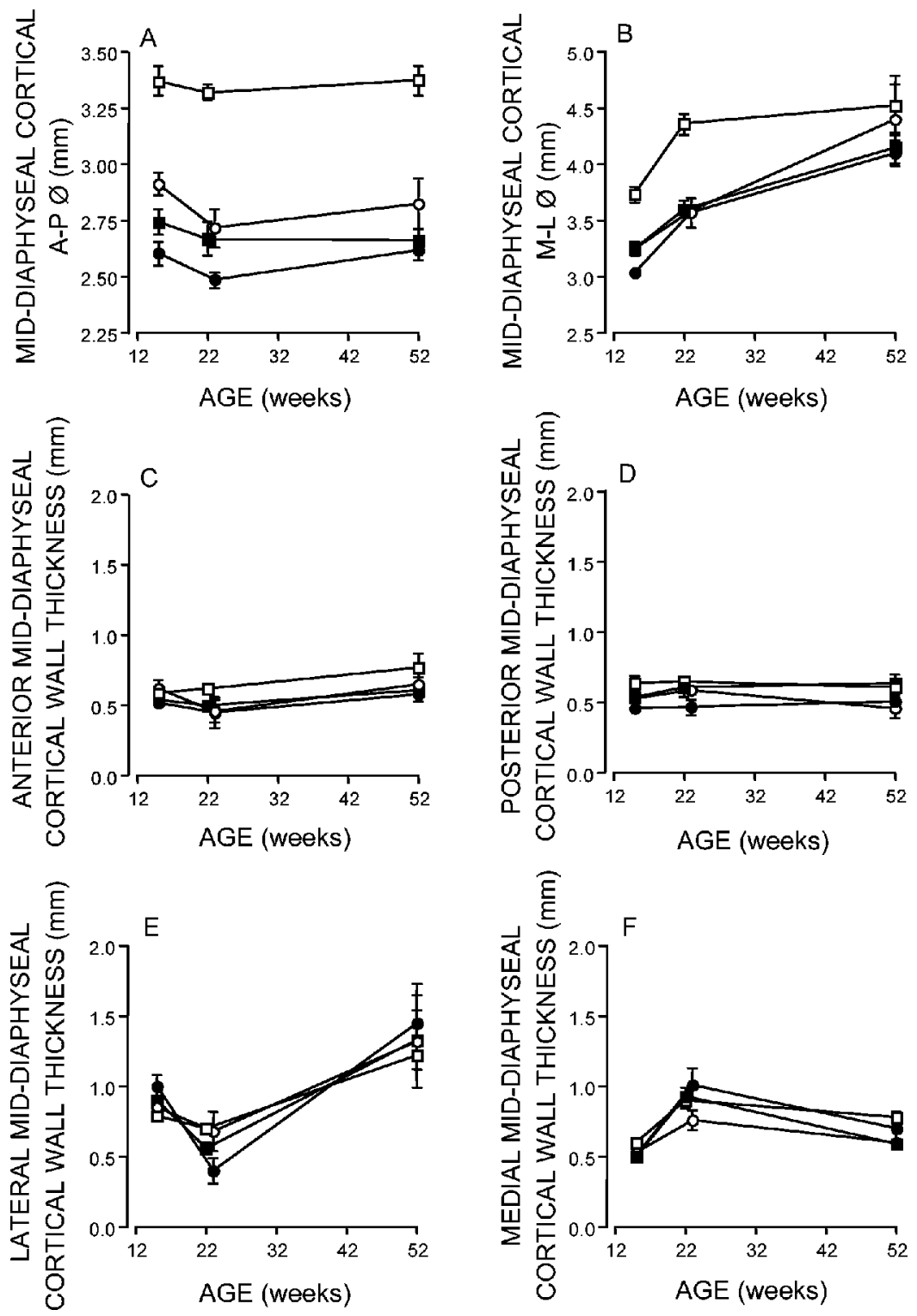

FIG. 3. The development of femoral morphology in male (squares) and female (circles) WT (open symbols) and Tgr (closed symbols) rats. Parameters shown are (A) mid-diaphyseal cortical anterior-posterior diameter (A-PØ), (B) middiaphyseal cortical medio-lateral diameter (M$\mathrm{L} \varnothing$ ), and (C) anterior, (D) posterior, (E) lateral, and $(\mathrm{F})$ medial mid-diaphyseal cortical wall thicknesses. Values shown are mean $\pm \mathrm{SE}(n=$ 4-6; and statistical comparisons described in the text made using one-way ANOVA and Bonferroni comparison).

the degree of trabecular anisotropy (an index of trabecular orientation) was significantly lower in femurs from Tgr rats $(p<0.01)$, indicating that the main axial direction is less pronounced in this model. Analysis of cortical bone from the distal femur showed little difference between WT and Tgr rats (data not shown).

\section{Plasma IGF-1 and leptin concentrations in Tgr rats}

Circulating IGF-1 declined with age in all groups but remained 4- to 7-fold higher in WT than Tgr males (Fig. 6A; $p<0.001)$. This difference was less pronounced in females, with plasma IGF-1 concentrations in 1-year-old Tgr rats being similar to their WT littermates (Fig. 6A). Plasma leptin concentrations remained largely unaltered in WT rats, with a small increase $(p<0.05)$ in WT females between 23 and 52 weeks (Fig. 6B). However, at 15 weeks, circulating leptin levels in Tgr males were 2-fold higher than all other groups $(p<0.001)$ and continued to rise steeply, remaining
3- to 4-fold higher than their WT counterparts at both 22 weeks $(p<0.01)$ and 1 year $(p<0.001)$. Plasma leptin concentration in Tgr females also rose steeply, in parallel with Tgr males and were double that of female WT littermates at $23(p<0.01)$ and 52 weeks $(p<0.001)$.

\section{DISCUSSION}

The regulation of cortical bone strength is achieved by a complex interaction between endocrine and mechanical factors controlling bone morphology and mineralization. As the principle endocrine regulator of postnatal longitudinal bone growth in mammals, $\mathrm{GH}$ is thought to act both directly through GH receptors in bone $e^{(20,21)}$ and indirectly through stimulation of the endocrine and paracrine actions of IGF-1. ${ }^{(22)}$ However, the influence of early onset GHD on bone strength in adulthood remains unclear. Although rats retain open epiphyseal growth plates into adulthood and are 

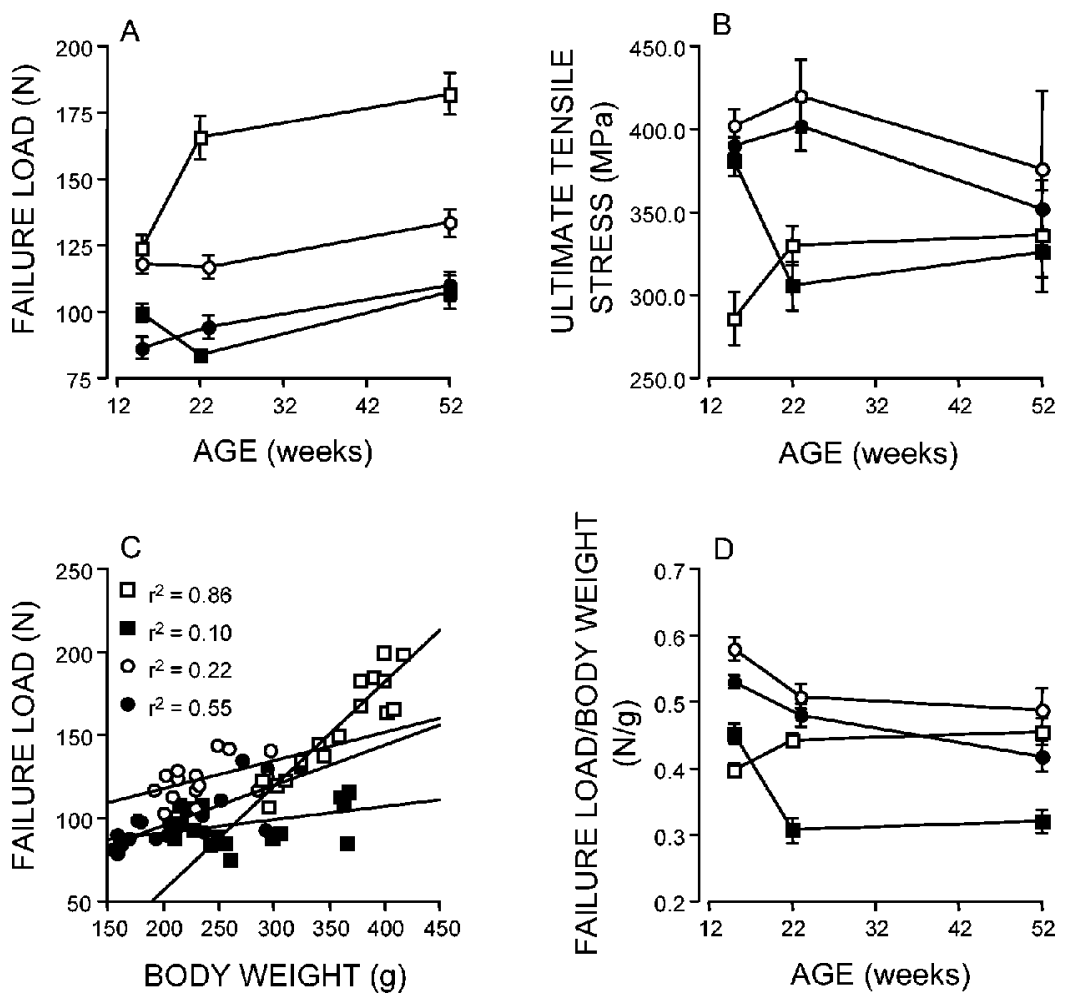

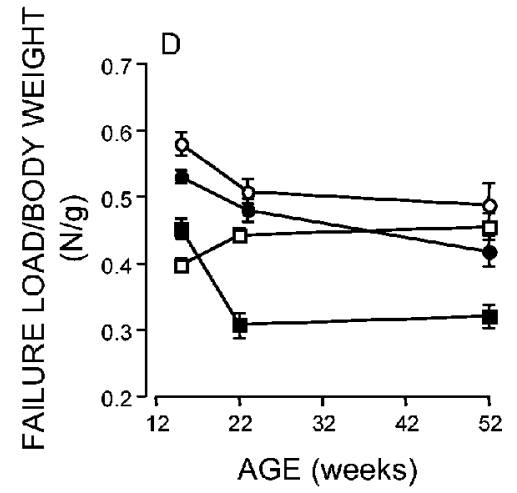

FIG. 4. The development of femoral strength in male (squares) and female (circles) WT (open symbols) and Tgr (closed symbols) rats. Parameters shown are (A) failure load, (B) ultimate tensile stress, $(C)$ regression analysis of failure load and body weight, and (D) the effect of adjustment of failure load for body weight. Values shown are mean $\pm \operatorname{SE}(n=4-6$; and statistical comparisons described in the text made using one-way ANOVA and Bonferroni or Dunnett's comparison as appropriate). not thought to undergo intracortical remodeling, ${ }^{(23)}$ many of the processes of bone growth are similar to those in humans. ${ }^{(13)}$ We have, therefore, used a new model of moderate GHD, the Tgr rat, to investigate the potential contribution of GH to the determinants of bone strength in early adulthood.

Our results indicate that $\mathrm{GH}$ is a significant determinant of bone strength, with a greater reduction in total femoral failure load in male Tgr rats (Fig. 4A) accompanying the greater degree of GHD in this sex. ${ }^{(15)}$ The effect of GH on bone strength does not seem to be mediated by its influence on body weight and the consequent alteration of load. Indeed, the relationship between body weight and failure load seen in normal male rats is disrupted in the Tgr model (Fig. 4C).

Bone strength is clearly related to bone size. ${ }^{(24)}$ In the Tgr model, the reduction in femoral length is accompanied by a sustained reduction in the external dimensions of the cortical bone, in particular the mid-diaphyseal cortical A-P diameter. Because failure load was determined by loading the bones in a posterior direction, the A-P diameter will have a greater impact on bone strength than the initial reduction in M-L diameter. In bending, the relationship between the tensile stresses produced and the distance from the neutral surface is approximately linear. Thus, the anterior and posterior surfaces carry high compressive and tensile stresses, respectively, while the medial and lateral surfaces bear only relatively small shear stresses. Cortical wall thickness, on the other hand, is not affected by the reduction in circulating $\mathrm{GH}$ in this model.

In addition to femoral geometry, the quality of bone tissue is an important determinant of strength. ${ }^{(24)}$ It was therefore surprising that, given the clearly observable differences in total femoral BMC and aBMD, calculation of
UTS revealed that after the rapid growth phase, the strength of the calcified tissue in Tgr rats was not significantly different. There may be a number of reasons for the disruption in the relationship between UTS and our measurements of femoral mineralization. First, tensile strength was only determined in the mid-diaphyseal cortex, whereas the measurements of BMD include the trabecular bone in the metaphyses. Second, using an areal technique to assess total femoral BMD only partially corrects for bone size, ${ }^{(25,26)}$ and may not produce an accurate representation of volumetric BMD at the cortical site of fracture. It should also be noted that the UTS values obtained in this study are somewhat higher than previously reported values for cortical bone in larger mammals. ${ }^{(23,27)}$ This may be accounted for by the different microstructure of cortical bone in rats and other small mammals. Rat cortical bone lacks both haversian systems and the weak points provided by the interconnecting cement lines and also exhibits little or no remodeling. ${ }^{(23)}$

The observation that UTSs are similar in Tgr and WT rats suggests that, after the rapid growth phase at least, GH does not have a significant impact on bone strength through its regulation of mineralization. This may not be so during the period of rapid growth. At 15 weeks, the earliest age measured in this study, UTS is, paradoxically, lower in WT males, which may be due, in part, to an increased rate of modeling/remodeling in this more rapidly growing cohort. As the growth rate slows in WT males between 15 and 22 weeks, there is a gradual increase in UTS. Conversely, the sharp decrease in UTS seen in Tgr males from 15 to 22 weeks may contribute to the declining failure load over the same period. The factors contributing to this decline are discussed below. 
Table 1. Trabecular Structure in 15-Week-Old WT and Tgr Male Rats

\begin{tabular}{lccccc}
\hline & $\begin{array}{c}\text { Relative bone density } \\
(\%)\end{array}$ & $\begin{array}{c}\text { Connectivity density } \\
\left(\text { connections } / \mathrm{mm}^{3}\right)\end{array}$ & $\begin{array}{c}\text { Trabecular thickness } \\
(\mathrm{mm})\end{array}$ & $\begin{array}{c}\text { Trabecular separation } \\
(\mathrm{mm})\end{array}$ & $\begin{array}{c}\text { Trabecular orientation } \\
\text { (degree of anisotropy) }\end{array}$ \\
\hline Wild-type male & $20.83 \pm 1.05$ & $83.26 \pm 2.89$ & $0.0842 \pm 0.0019$ & $0.298 \pm 0.018$ & $1.704 \pm 0.024$ \\
Tgr male & $14.12 \pm 1.04^{\dagger}$ & $67.69 \pm 3.74^{*}$ & $0.0753 \pm 0.0024^{*}$ & $0.400 \pm 0.009^{\dagger}$ & $1.453 \pm 0.026^{\dagger}$ \\
\hline
\end{tabular}

Values shown are mean $\pm \mathrm{SE}(n=3$; statistical comparisons made using Student's $t$-test).

$* p<0.05$

${ }^{\dagger} p<0.01$.

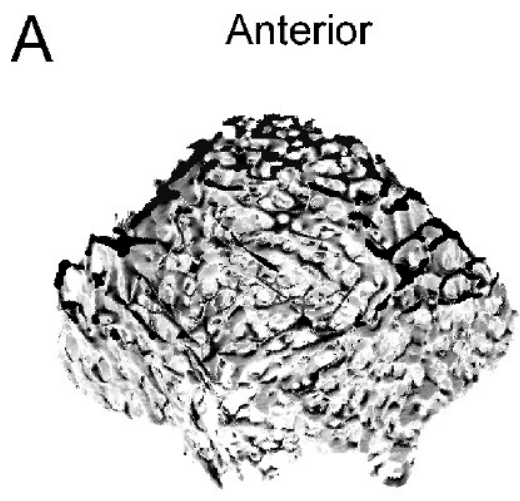

Posterior

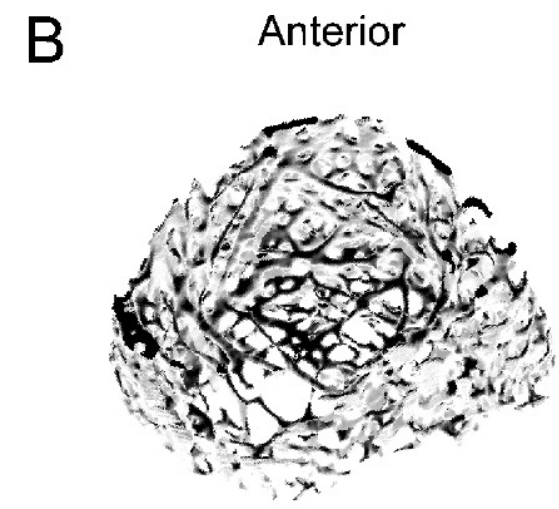

Posterior
FIG. 5. Micro-CT 3-D reconstructions of trabecular structure in distal femur from (A) WT and (B) Tgr male femurs. Each image represents an anterior-posterior cross-section through the distal femur adjacent to the growth plate, viewing the trabecular organization from the growth plate toward the shaft. The reduction in trabecular number and increase in trabecular separation that give rise to the reduction in relative bone density in Tgr rats (Table 1) are clearly observable in these images.

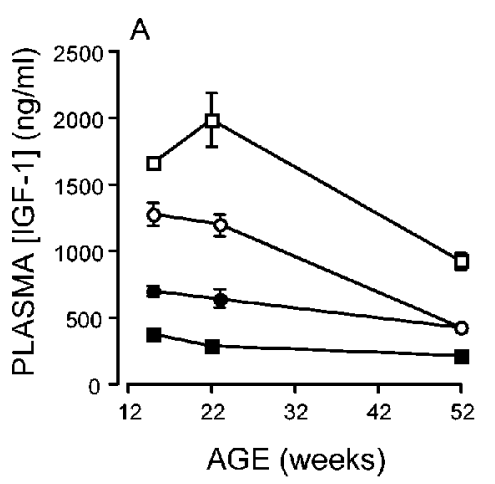

While changes in trabecular microachitecture are unlikely to contribute to the reduction in cortical strength in the Tgr rat, such alterations provide a helpful insight into the process of remodeling. The combination of fewer trabeculae with greater heterogeneity of trabecular thickness and less pronounced main axial direction indicates that, in the context of moderate GHD, the control of trabecular organization is altered. A reduction in tibial trabecular bone volume has previously been reported for the profoundly GHD $d w / d w$ rat. ${ }^{(28)}$ The presence of smaller numbers of more widely spaced trabeculae in GHD rats is consistent with remodeling simulations, ${ }^{(29,30)}$ predicting that resorption results in loss of trabeculae even with subsequent bone deposition. Should new bone deposition be inhibited, not only would trabecular number remain reduced, but the positive correlation between external load (body weight) and bone strength will be disrupted. This is the case in Tgr males, where the positive correlation between body weight and failure load seen in WT males is absent (Fig. 4C).

Thus, the influence of GH on femoral strength seems to be caused by its effects on bone size rather than on bone quality. Similar findings have been reported for MT-GHtransgenic mice with elevated circulating GH concentrations, in which increased mechanical strength is associated with increased cortical width ${ }^{(31,32)}$ and also in 2-year-old male rats treated with hGH for up to 80 days. ${ }^{(33)}$ However, our experiments do not distinguish between the direct effects of $\mathrm{GH}$ and those mediated by IGF-1. The results reported here confirm that circulating IGF-1 concentrations are profoundly reduced in this model, ${ }^{(15)}$ and show that, as with their WT littermates, IGF-1 production declines with age (Fig. 6A). However, these measurements may not provide an accurate reflection of IGF-1 bioavailability. This is influenced by the circulating IGF-binding proteins, which 
were not measured in this study. In growing children circulating IGF-1 is a major determinant of the cross-sectional morphology of the femur, but has no influence on the material density of cortical bone. ${ }^{(34)}$

The development of elevated circulating leptin concentrations in the Tgr rat is an important new observation in the context of the reported influences of this hormone on bone. ${ }^{(35,36)}$ The steep rise in circulating leptin seen in Tgr rats is likely to relate to the increased visceral fat deposition in this model (our unpublished data), which may contribute to the converging body weights when there is no corresponding convergence in skeletal size. Thus, the increased contribution of fat deposition to body weight and the resultant elevation in circulating leptin may contribute to the disruption of the relationship between body weight and failure load.

It is now thought that leptin has a range of actions on bone, ${ }^{(37)}$ although some of the published findings appear to be conflicting. For example, while leptin stimulates bone growth in $o b / o b$ mice, ${ }^{(36)}$ it also inhibits bone formation. ${ }^{(35)}$ It is proposed that this latter action involves a hypothalamic component. ${ }^{(35)}$ However, the high levels of leptin expression in bone marrow adipocytes, ${ }^{(38)}$ and the action of leptin to favor the differentiation of marrow stromal cells into osteoblasts rather than adipocytes in vitro, ${ }^{(39)}$ suggest an autocrine/paracrine action for leptin. Thus, the development of elevated circulating leptin concentrations in Tgr rats may have a profound impact on the factors regulating bone strength in this model, particularly if the mechanism favoring elevated visceral adiposity and circulating leptin enhances adipogenesis and leptin expression in marrow. It is possible that the steep rise in circulating leptin concentration contributes to the observed decline in strength of both the calcified material (UTS; Fig. 4B) and the whole femur (failure load; Fig. 4A) between 15 and 22 weeks in male Tgr rats, particularly in the context of an $85 \%$ reduction in circulating IGF-1. This effect of leptin may be obscured in Tgr females by the presence of higher levels of circulating GH. ${ }^{(15)}$

The possible impact of leptin on the endocrine control of the bone microenvironment in this model has implications for the potential therapeutic modalities used to accelerate skeletal growth. A unique feature of the Tgr model of dwarfism is that the residual responsiveness of the hypothalamo-pituitary $\mathrm{GH}$ axis to treatment with the $\mathrm{GH}$ secretagogues (GHSs) ${ }^{(15)}$ is sufficient to accelerate skeletal growth. ${ }^{(40)}$ However, it is now clear that ghrelin, the endogenous ligand for the GHS receptor, ${ }^{(41)}$ induces adiposity in the context of GHD. ${ }^{(42)}$ While we are currently investigating the adipogenic effect of the GHSs in the Tgr model, these findings suggest that optimal growth recovery may be obtained with more conventional GH replacement.

In summary, the Tgr rat is a model of moderate GHD, in which reduced plasma IGF-1 and increased circulating leptin are accompanied by a profound reduction in femoral strength. The weakness of the femoral cortex appears to be more closely related to femoral diameter than to the material properties of the calcified tissue. The disruption of the relationship between body weight and failure load in this model indicates that the effect of GH on bone strength is not mediated by its influence on body weight. In addition, these results suggest that patients with moderate childhood onset GHD may have an increased risk of fracture if left untreated during their growth phase and that fracture risk may be further increased if GHD is accompanied by elevated adiposity.

\section{ACKNOWLEDGMENTS}

The authors thank Dr Debbie Mason (Cardiff University) for helpful suggestions, and John Tobias and Mark Perry (Bristol University, UK) for use of Lunar Pixi Scanner. In addition, JTW acknowledges financial support from the British Society for Paediatric Endocrinology and Diabetes, Pharmacia \& Upjohn, and the Welsh Office of Research and Development for Health and Social Care, and TW acknowledges the financial support of the Medical Research Council (UK; Grant G9625574N) and the Biotechnology and Biosciences Research Council (UK; Grant 72/S11914).

\section{REFERENCES}

1. Bouillon R 1991 Growth hormone and bone. Horm Res 36:49-55. 2. Ohlsson C, Bengtsson B-Å, Isaksson OGP, Andreasson TT, Slootewg MC 1998 Growth hormone and bone. Endocr Rev 19: 55-79.

3. Holmes SJ, Economou G, Whitehouse RW, Adams JE, Shalet SM 1994 Reduced bone mineral density in patients with adult onset growth hormone deficiency. J Clin Endocrinol Metab 78:669-674.

4. Rosén T, Wilhelmsen L, Landin-Wilhelmsen K, Lappas G, Bengtsson B- 1997 Increased fracture frequency in adult patients with hypopituitarism and GH deficiency. Eur J Endocrinol 137:240245.

5. Raine JE, Donaldson MDC, Gregory JW, Savage MO 2001 Short stature. In: Practical Endocrinology and Diabetes in Children. Blackwell Science, Oxford, UK, pp. 37-60.

6. Growth Hormone Research Society 2000 Consensus guidelines for the diagnosis and treatment of growth hormone $(\mathrm{GH})$ deficiency in childhood and adolescence: Summary statement of the GH Research Society. J Clin Endocrinol Metab 85:3990-3993.

7. Guyda HJ 2000 Growth hormone testing and the short child. Pediatr Res 48:579-580.

8. Shore RM, Chesney RW, Mazess RB, Rose PG, Bargman GJ 1980 Bone mineral status in growth hormone deficiency. J Pediatr 96: 393-396.

9. Kaufman JM, Taelman P, Vermeulen A, Vandeweghe M 1992 Bone mineral status in growth hormone-deficient males with isolated and multiple pituitary deficiencies of childhood onset. J Clin Endocrinol Metab 74:118-123.

10. Hyer SL, Rodin DA, Tobias JH, Leiper A, Nussey SS 1992 Growth hormone deficiency during puberty reduces adult bone mineral density. Arch Dis Child 67:1472-1474.

11. Lissett CA, Shalet SM 2000 Effects of growth hormone on bone and muscle. Growth Horm IGF Res 10(Suppl B):S95-S101.

12. Cowell CT, Woodhead HJ, Brody J 2000 Bone markers and bone mineral density during growth hormone treatment in children with growth hormone deficiency. Horm Res 54(Suppl 1):44-51.

13. Frost HM, Jee WS 1992 On the rat model of human osteopenias and osteoporoses. Bone Miner 18:227-236.

14. Flavell DM, Wells T, Wells SE, Carmignac DF, Thomas GB, Robinson ICAF 1996 A new dwarf rat: Dominant negative phenotype in GRF-GH transgenic growth retarded (Tgr) rats. EMBO J 15:3871-3879.

15. Wells T, Flavell DM, Wells SE, Carmignac DF, Robinson ICAF 1997 Effects of GH secretagogues in the transgenic growth retarded (Tgr) rat. Endocrinology 138:580-587.

16. Charlton HM, Clark RG, Robinson ICAF, Porter Goff AE, Cox BS, Bugnon C, Bloch BA 1988 Growth hormone-deficient dwarfism in the rat: A new mutation. J Endocrinol 119:51-58.

17. Samuels A, Perry MJ, Gibson RL, Colley S, Tobias JH 2001 Role of endothelial nitric oxide synthase in estrogen-induced osteogenesis. Bone 29:24-29.

18. Laib A, Barou O, Vico L, Lafage-Proust MH, Alexandre C, Rüegsegger P 2000 3D micro-computed tomography of trabecular and 
cortical bone architecture with application to a rat model of immobilisation osteoporosis. Med Biol Eng Comput 38:326-332.

19. Hildebrand T, Rüegsegger P 1997 A new method for the model independent assessment of thickness in three-dimensional images. J Microsc 185:67-75.

20. Isaksson OG, Jansson JO, Gause IA 1982 Growth hormone stimulates longitudinal bone growth directly. Science 216:1237-1239.

21. Baker AR, Hollingshead PG, Pitts-Meek S, Hanson S, Taylor R, Stewart TA 1992 Osteoblast-specific expression of growth hormone stimulates bone growth in transgenic mice. Mol Cell Biol 12:5541-5547.

22. Isaksson OG, Lindahl A, Nilsson A, Isgaard J 1987 Mechanism of the stimulatory effect of growth hormone on longitudinal bone growth. Endocr Rev 8:426-438.

23. Currey J 1982 The mechanical properties of materials and the structure of bone, and the mechanical properties of bone. In: The Mechanical Adaptations of Bone. Princeton University Press, Princeton, NJ, USA, pp. 3-87.

24. Van der Meulen MCH, Jepsen KJ, Miki'c B 2001 Understanding bone strength: Size isn't everything. Bone 29:101-104.

25. Carter DR, Bouxsein ML, Marcus R 1992 New approaches for interpreting projected bone densitometry data. J Bone Miner Res 7:137-145.

26. Seeman E 1998 Growth in bone mass and size-are racial and gender differences in bone mineral density more apparent than real? J Clin Endocrinol Metab 83:1414-1419.

27. Keaveney TM, Hayes WC 1993 A twenty year perspective on the mechanical properties of trabecular bone. Trans ASME. J Biomech Eng 115:534-542.

28. Wright NM, Renault J, Hollis B, Bell NH, Ley LL 1995 Effect of growth hormone on bone: Bone mineral density, trabecular bone volume, and alkaline phosphatase improve, or are restored in the dwarf rat treated with growth hormone. J Bone Miner Res 10:127131.

29. Mullender MG, Huiskes RA 1995 A proposal for the regulatory mechanism of Wolff's law. J Orthrop Res 13:503-512.

30. Huiskes R, Ruimerman R, van Lenthe GH, Janssen JD 2000 Effects of mechanical forces on maintenance and adaptation of form in trabecular bone. Nature 405:704-706.

31. Wanke R, Wolf E, Hermanns W, Folger S, Buchmuller T, Brem G 1992 The GH-transgenic mouse as an experimental model for growth research: Clinical and pathological studies. Horm Res 37:74-87.

32. Ohlsson C, Tornell J, Sandstedt J, Andraessen TT 1996 Effect of increased growth hormone on mechanical strength of cortical bone in growth hormone-transgenic mice. J Bone Miner Res 11:S1; S458.

33. Andreasson TT, Jørgenssen PH, Flyvbjerg A, Ørskov H, Oxlund H 1995 Growth hormone stimulates bone formation and strength of cortical bone in aged rats. J Bone Miner Res 10:1057-1067.
34. Mora S, Pitukcheewanont P, Nelson JC, Gilsanz V 1999 Serum levels of insulin-like growth factor I and the density, volume and cross-sectional area of cortical bone in children. J Clin Endocrinol Metab 84:2780-2783.

35. Ducy P, Amling M, Takeda S, Priemel M, Schilling AF, Beil FT, Shen J, Vinson C, Rueger JM, Karsenty G 2000 Leptin inhibits bone formation through a hypothalamic relay: A central control of bone mass. Cell 100:197-207.

36. Steppan CM, Crawford DT, Chidsey-Frink KL, Ke HZ, Swick AG 2000 Leptin is a potent stimulator of bone growth in $o b / o b$ mice. Regul Pept 92:73-78.

37. Gordeladze JO, Reseland JE, Drevon CA 2001 Pharmocological interference with transcriptional control of osteoblasts: A possible role for leptin and fatty acids in maintaining bone strength and body lean mass. Curr Pharm Des 7:275-290.

38. Laharrague P, Larrouy D, Fontanilles A-M, Truel N, Campfield A, Tenenbaum R, Galitzky J, Corberand JX, Pénicaud L, Casteilla L 1998 High expression of leptin by human bone marrow adipocytes in primary culture. FASEB J 12:747-752.

39. Thomas T, Gori F, Khosla S, Jensen MD, Burguera B, Riggs BL 1999 Leptin acts on human marrow stromal cells to enhance differentiation to osteoblasts and to inhibit differentiation to adipocytes. Endocrinology 140:1630-1638.

40. Wells T, Houston PA 2001 Skeletal growth acceleration with growth hormone secretagogues in transgenic growth retarded (Tgr) rats: Pattern-dependent effects and mechanisms of desensitization. J Neuroendocrinol 13:496-504.

41. Kojima M, Hosoda H, Date Y, Nakazato H, Kangawa K 1999 Ghrelin is a growth hormone-releasing acylated peptide from stomach. Nature 402:656-660.

42. Tschöp M, Smiley DL, Heinman ML 2000 Ghrelin induces adiposity in rodents. Nature 407:908-913.

Address reprint requests to: T Wells, BSc, PhD

School of Biosciences

Cardiff University

PO Box 911

Museum Avenue

Cardiff CF10 3US, UK

E-mail:wellst@cardiff.ac.uk

Received in original form August 6, 2002; in revised form December 20, 2002; accepted January 15, 2003. 